\title{
Determining an optimal management strategy for upper airway obstruction in Pierre Robin sequence
}

\author{
Sherri Lynne Katz MDCM MSc FRCPC
}

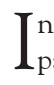

In the current issue of the Journal, Kam et al (pages 171-175) compare two institutions' experiences using multiple intervention strategies in the management of upper airway obstruction in infants with Pierre Robin sequence. They used a retrospective descriptive design to examine characteristics and outcomes in 139 children evaluated over one decade. Interventions ranged from minimally invasive (prone positioning [61\%]) to extremely invasive (tracheostomy [19\%]). This study adds to the existing body of literature in this population, which has largely reported case series using multiple interventions with varying success. Previous studies may have suffered from bias in the selection of patients for a particular intervention and did not have comparison groups other than conservative management. Few considered continuous positive airway pressure (CPAP) as a treatment option $(1,2)$. This study was able to compare the outcomes of different treatment modalities, albeit without a standardized approach to choice of intervention. Low birth weight and associated syndromes were identified as determinants of the need for tracheostomy. Nonetheless, Kam et al concluded that intervention choice remains "clinician and resource dependent". The lack of a standardized methodology for work-up and intervention leaves many unanswered questions about how to best approach the care of such children.

There are, unfortunately, limitations to the data obtained from this study, which preclude its use to confidently predict optimal treatment for an individual. Selection bias likely occurred, in that those who were sicker initially or had other comorbidities may have received earlier or more aggressive intervention. Although this type of bias could only be eliminated with a randomized controlled trial, this may have affected some of the reported outcomes. For instance, children requiring surgical intervention of any kind had longer intensive care unit stays than those who underwent nonsurgical treatment. Beyond the obvious requirements for postoperative recovery, other clinical factors, such as severity of upper airway obstruction, which are inconsistently available on retrospective review, may have influenced length of stay or choice of intervention. It also remains unclear whether earlier aggressive intervention (ie, tracheostomy or other surgery) led to better clinical outcomes and whether a delay in initial intervention was detrimental. These key pieces of information cannot be gleaned from this study, but are critical to inform best practices.

A second challenge in the interpretation of these results relates to the utility of polysomnography (PSG), indicated by Kam et al as important to guide clinical decision making. In this study, very few patients underwent PSG, making it difficult to draw conclusions on the value of such testing. PSG was only performed in $30 \%$ of patients, with only $12 \%$ of PSGs being performed before intervention. Most children underwent PSG at a later age (mean 23 months). Yet, of those who underwent PSG, CPAP was used in a higher proportion of patients than other treatments and one-quarter required no further intervention. This suggests that CPAP - a less invasive treatment than tracheostomy and other surgical treatments - can be initiated and its impact evaluated during the course of PSG. Perhaps earlier PSG in this population would result in more CPAP treatment initiation and success in a greater proportion of patients, a strategy used in other case series $(1,2)$. It is possible that earlier consideration of CPAP therapy may, in some cases, obviate the need for more invasive therapies including tracheostomy. Furthermore, use of PSG for identification of children who do not require further intervention may save some from unnecessary treatments. This study cannot answer the question of whether PSG before intervention would have changed clinical practice, particularly because very few children (six of 19) who had a tracheostomy underwent PSG.

The limited use of PSG before intervention in this study likely reflects changing trends in health care over the study period, as well as the limited availability of this resource (3). PSG is now recognized as the gold standard test for diagnosis of sleep-disordered breathing (4) and is recommended in the evaluation of children with craniofacial anomalies $(1,2)$, particularly because significant hypoxemia and apnea may be present even in the absence of clear symptoms of airway obstruction $(5,6)$. Overnight oximetry recordings or modified PSG may present an alternative where PSG is unavailable $(2,4,7,8)$.

Thus, while this study expands our knowledge of clinical factors predicting need for earlier intervention in children with Pierre Robin sequence, it does highlight the significant variability in treatments for this population and demonstrates that additional data are needed to guide clinical practice. This provides good rationale to conduct prospective studies to determine best practices and aid clinical decision making, including definition of the role of PSG and oximetry. A prospective study will also enable more standardized data collection, which will contribute to an enhanced understanding of risk factors determining the need and optimal timing for particular interventions. Ultimately, a clinical algorithm to guide best practices may be developed.

\section{REFERENCES}

1. Daniel M, Bailey S, Walker K, et al. Airway, feeding and growth in infants with Robin sequence and sleep apnoea. Int J Pediatr Otorhinolaryngol 2013;77:499-503.

2. van Lieshout MJ, Joosten KF, Hoeve HL, Mathijssen IM, Koudstaal MJ, Wolvius EB. Unravelling Robin sequence: Considerations of diagnosis and treatment. Laryngoscope 2014;124:E203-E209.

3. Katz SL, Witmans M, Barrowman N, et al. Paediatric sleep resources in Canada: The scope of the problem. Paediatr Child Health 2014;19:367-72.

4. Marcus CL, Brooks LJ, Draper KA, et al. Diagnosis and management of childhood obstructive sleep apnea syndrome. Pediatrics 2012;130:576-84.

5. Anderson IC, Sedaghat AR, McGinley BM, Redett RJ, Boss EF, Ishman SL. Prevalence and severity of obstructive sleep apnea and snoring in infants with Pierre Robin sequence. Cleft Palate Craniofac J 2011;48:614-8.

6. MacLean JE, Fitzsimons D, Fitzgerald DA, Waters KA. The spectrum of sleep-disordered breathing symptoms and respiratory events in infants with cleft lip and/or palate. Arch Dis Child 2012;97:1058-63.

7. Bull MJ, Givan DC, Sadove AM, Bixler D, Hearn D. Improved outcome in Pierre Robin sequence: Effect of multidisciplinary evaluation and management. Pediatrics 1990;86:294-301.

8. Abel F, Bajaj Y, Wyatt M, Wallis C. The successful use of the nasopharyngeal airway in Pierre Robin sequence: An 11-year experience. Arch Dis Child 2012;97:331-4. 


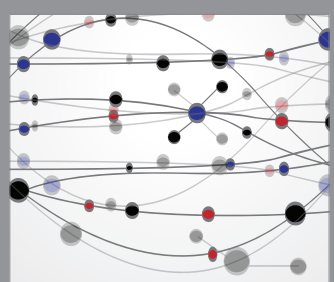

The Scientific World Journal
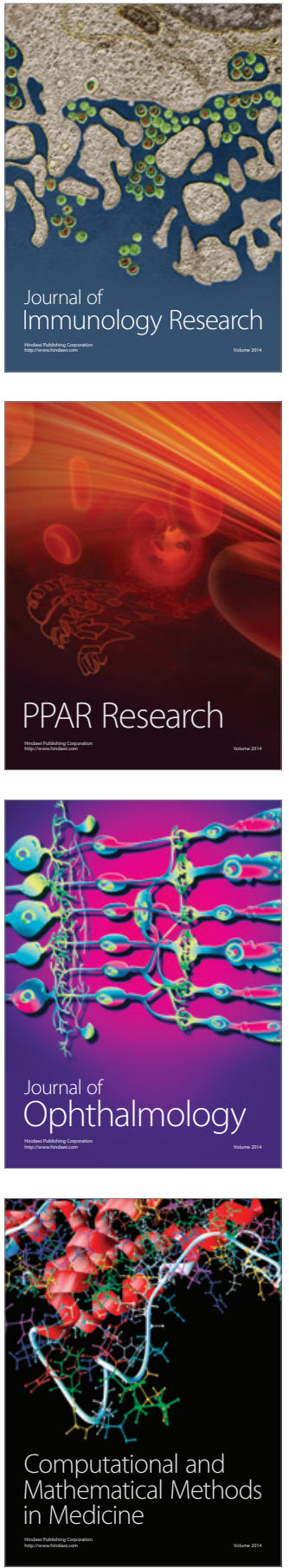

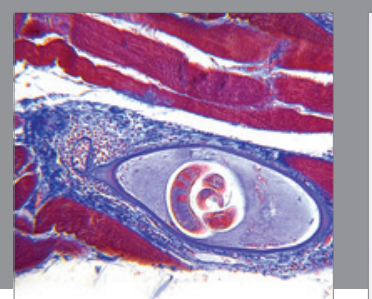

Gastroenterology Research and Practice

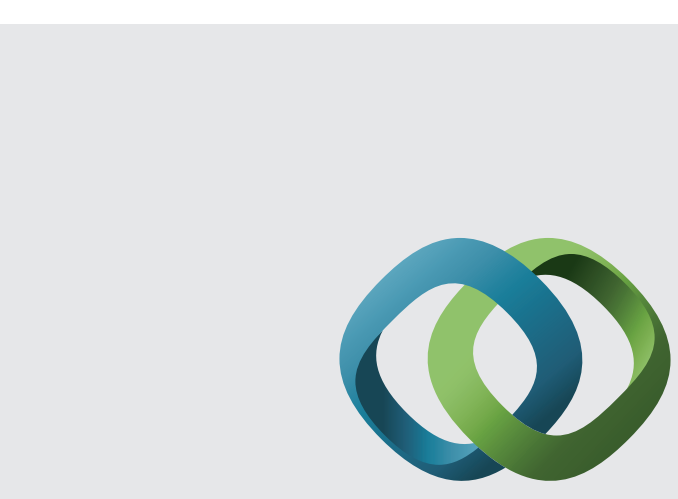

\section{Hindawi}

Submit your manuscripts at

http://www.hindawi.com
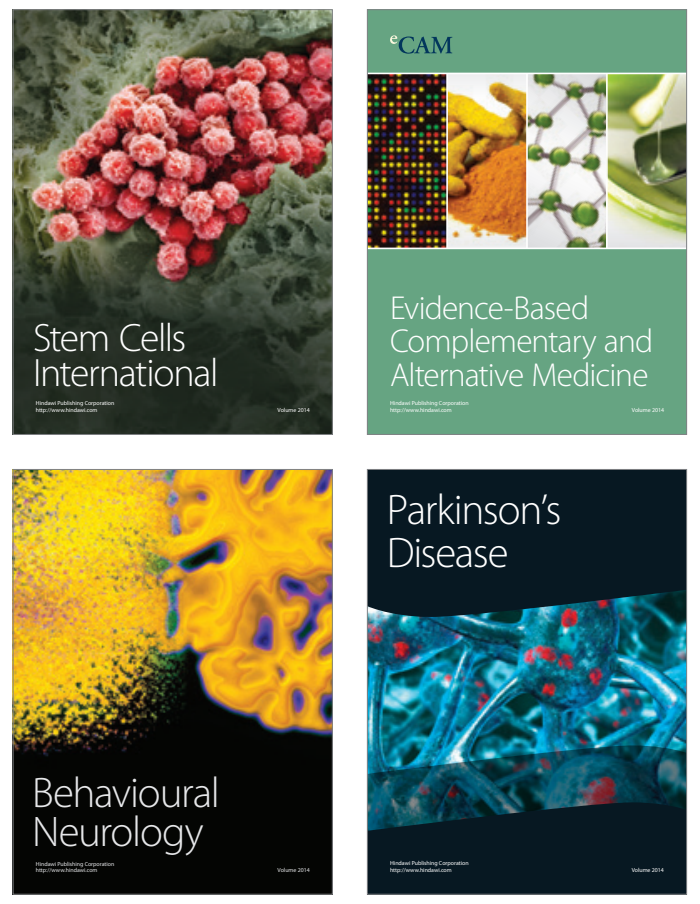
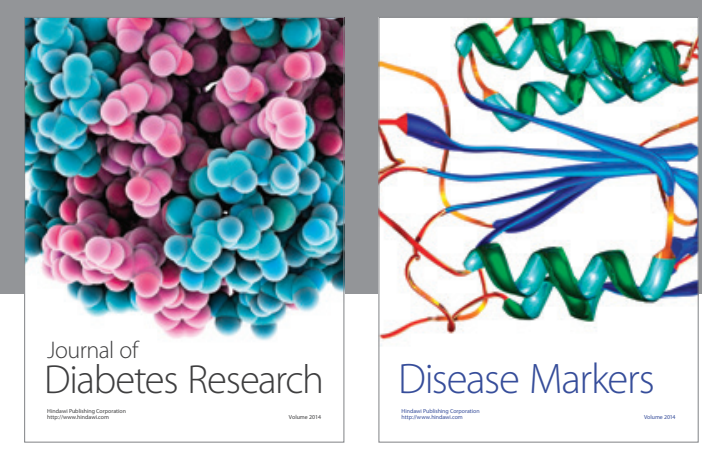

Disease Markers
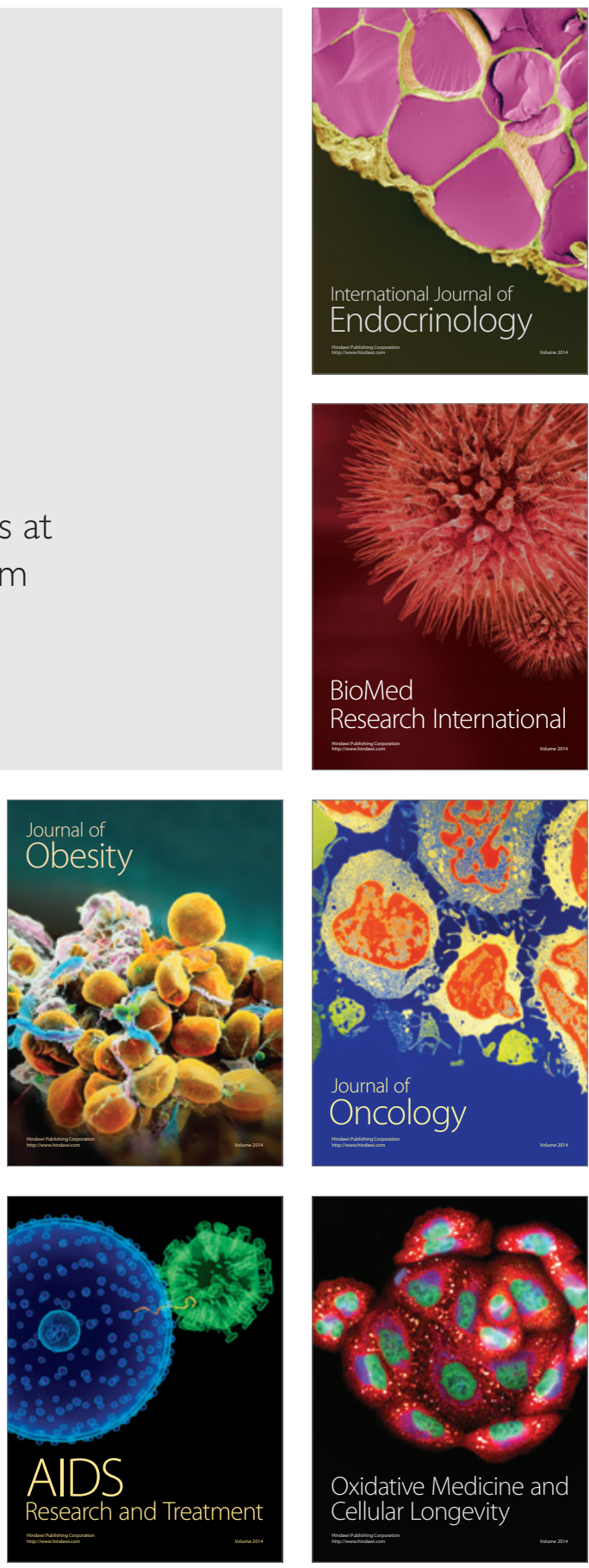